\title{
Sistema SCADA UNAH para eficiencia energética
}

\author{
Dennis A. Rivera ${ }^{1}$ \\ José Gabriel Zorto Aguilera ${ }^{2}$
}

\section{RESUMEN}

Un sistema SCADA es una aplicación de software especializada para funcionar sobre computadoras y dispositivos de campo, para el monitoreo y control de cualquier sistema o instalación a distancia; es por esta razón que se presenta una propuesta para la implementación de un sistema SCADA en la UNAH, la que tiene como principal objetivo reducir los costos en la factura por consumo de energía eléctrica, ya que la universidad actualmente cuenta con un alto consumo energético. Además la misma universidad dispone de un anillo de fibra óptica que está presente en los predios universitarios, (donde el ente responsable del manejo de dicha red es la Dirección Ejecutiva de Gestión de Tecnología DEGT) el cual reduce los costos de manera importante; es una razón de vital importancia para llevar a cabo este proyecto.

El sistema SCADA, nos permitirá monitorear y controlar los dispositivos instalados (Tarjeta de adquisición de datos, PC, PLC, y los diferentes sensores de movimiento y capacitivos), donde éstos a su vez se interconectan a la Red VLAN por medio de un Switch, y con los demás edificios a través del anillo de fibra óptica, el cual tiene su servidor principal.

Es importante hacer mención que la misma Investigación nos llevó a realizar un prototipo de SCADA REAL de bajo costo, para lograr evidenciar el funcionamiento de este tipo de sistema y demostrar que es posible aplicarlo a la UNAH. El Prototipo fue desarrollado utilizando una tarjeta de Adquisición de Datos, Proyect Board, y dispositivos electrónicos, realizando la toma de datos del mundo real y el control de estas variables físicas a través de computadoras remotas por medio de una red de comunicación Wireless, simulando una Red LAN TCP/IP. El proyecto y el prototipo fueron presentados oralmente en la clase de Seminario de investigación y de forma muy especial también a la DEGT, donde se formuló la implementación del SCADA arrancando con un edificio piloto para la elaboración y desarrollo del sistema para llevarlo a toda laUNAH.

\footnotetext{
${ }^{1}$ Catedrático Seminario de Investigación IE-900, UNAH.

${ }^{2}$ Estudiante de Ingeniería Eléctrica.
} 
El plan de inversión para los 25 edificios de alto consumo de energía eléctrica de la UNAH, es de L. 3,903,743.75, donde los fondos podrían ser adquiridos de la siguiente forma: $30 \%$ fondos propios o provenientes de fuentes externas, L.1 $171,123.13$ y un $70 \%$ financiado por una 0 varias entidades financieras L. 2 732,620.63. Estas inversiones incluyen mobiliario, software y hardware únicamente. Los costos pueden variar considerando, que mucho equipo de Red ya lo posee la DEGT, por ello es importante que el detalle de estos mismos los brinde la DEGT y sea ella misma la que defina el costo final.

En las condiciones en que se plantea el proyecto, nos permite recuperar la inversión en un año y seis meses como se muestra de forma paralela la relación que existe entre el beneficio y el costo de la implementación del proyecto, ya que el beneficio es muy rentable para más de cinco años. Ver Fig.8

Debido a la magnitud del proyecto es sumamente importante contratar a un profesional de la ingeniería que labore en la DEGT con dedicación exclusiva, con amplios conocimientos de electricidad, software, autómatas y Redes, otorgándole facultades suficientes para coordinar y gestionar la implementación y desarrollo de este proyecto y de los restantes proyectos de ahorro energético en los edificios de la UNAH. De implementarse el proyecto de forma completa, el ahorro económico que tendría la UNAH seria de más de un millón de lempiras anualmente con tan sólo disminuir una hora de iluminación diaria.

Palabras Clave: SCADA, Automatización, Redes y Protocolos de comunicación, Control Remoto, Informática de Sistemas de Automatización y Control.

\section{SUMMARY}

A SCADA system is a specialized software application to run on computers and field devices for monitoring and control of any system or distant installations. Therefore, the following is a proposal for the implementation of a SCADA system in the UNAH, which has as a main objective the reduction of electricity consumption costs, since the university currently has high energy consumption. In addition, the university has a fiber optic ring that is present on campus (where the entity responsible for managing the network is the Executive Director of Technology Management EDTM, DEGT in Spanish), which reduces the initial investment needed, and is a vital reason for conducting this project. 
The SCADA system will enable the user to monitor and control installed devices (data acquisition card, PC, PLC, and various motion and capacitive sensors), where these are interconnected to the network via a VLAN through a Switch and with the other buildings through fiber optic ring, which has its main server.

It is important to mention that the same research led us to perform a REAL SCADA prototype of low cost, to demonstrate the performance of this type system and prove that it can be applied to the UNAH. The prototype was developed using a Data Acquisition Card, Project Board, and electronic devices, Performing the real-world data acquisition and control of these physical variables through remote computers via a wireless communication network, for simulation of a network LAN TCP / IP. The design and prototype were presented orally at the Research Seminar class and specifically to DEGT, where development and implementation of SCADA were explained, which must start with a pilot Building for the design and development of the system to carry all UNAH.

The investment plan for the 25 buildings with high consumption of electrical power is $3,903,743.75$ LPS, where funds could be acquired as follows, $30 \%$ own funds or from external sources $(1,171,123.13$ LPS), and $70 \%$ funded by one or more financial institutions $(2,732,620.63$ LPS). These investments include furniture, software and hardware only, these costs can vary considerably, because much of the network equipment already exist in university. The DEGT manages these resources, so it is important that, the detail of these must given by them and is itself which defines the final cost.

The conditions in which the project arises, allows us to recover the investment in eighteen months. As shown in parallel the relationship between benefit and cost of project implementation, because the benefit is very profitable for more than five years. See Fig. 8

Due to the magnitude of the project, it is extremely important to hire a professional engineer to work in DEGT full time, with extensive knowledge of electricity, software, automated systems and networks. Granting sufficient authority to coordinate and manage the implementation and development of this project and other energy saving projects in buildings of the National Autonomous University of Honduras. If the complete project is implemented, the economic savings that the UNAH would have is more than a million Lempiras annually.

\section{Key words: SCADA, Automation, Networks and Communication Protocols, Remote Control, Computer Systems for Automation and Control.}




\section{INTRODUCCIÓN}

La tecnología evoluciona a diario, con ello se generan oportunidades de mejora en diversas áreas, para disminuir tiempo en los procesos y reducción de costos, entre otros.

El presente documento detalla una propuesta que permite precisamente el uso de la tecnología para la reducción de costos fijos dentro de las organizaciones; específicamente la implementación de un sistema SCADA para controlar el consumo de energía eléctrica en la Universidad Nacional Autónoma de Honduras.

El establecimiento del problema de investigación, es la reducción del consumo irracional de energía. El problema de investigación se respalda con la importancia de tomar acciones para la reducción de la factura energética.

Encontramos el estudio técnico que incluye planos que detallan la red de comunicación, las instalaciones eléctricas de los edificios y por ende la localización del proyecto. También se detallan las características de cada uno de los equipos y software que se requieren.

Como parte de la investigación se desarrollo un prototipo de Mini-SCADA con el propósito de enlazar el mundo real y virtual realizando adquisición de información del mundo real y controlando las variables físicas a través de la interfaz por PC. Con el desarrollo de este pequeño SCADA dejamos demostrado que podemos desarrollar y tener a nuestro alcance este tipo de tecnología.

También mostramos el estudio económico donde presenta el monto de la inversión necesaria para la ejecución del proyecto, junto con las fuentes de financiamiento que podrían utilizarse para cubrir la inversión. Al mismo tiempo se cuantifica, en dinero, el ahorro que se generaría al implementar el proyecto; finalmente se presentan los flujos de efectivos, los cuales permiten calcular el valor presente neto (VPN) y la tasa interna de retorno (TIR) que se obtendrían.

En virtud de la importancia que tiene la eficiencia energética, se presenta esta propuesta metodológica, que al poseer un sistema de tal magnitud, nos permitirá estar a la vanguardia de la tecnología, y así poder estimular a la comunidad universitaria a realizar proyectos que permitan obtener beneficios en diferentes áreas. 


\section{SISTEMASCADA}

\section{Definición}

SCADA viene de las siglas de "Supervisory Control And Data Adquisition", es decir: adquisición de datos y control de supervisión. Se trata de una aplicación software especialmente diseñada para funcionar sobre ordenadores en el control de producción, proporcionando comunicación con los dispositivos de campo (controladores autónomos, autómatas programables, etc.) y controlando el proceso de forma automática desde la pantalla del ordenador.

Además, provee de toda la información que se genera en el proceso productivo a diversos usuarios, tanto del mismo nivel como de otros supervisores dentro de la UNAH: control de calidad, supervisión y mantenimiento, etc.

\section{Componentes de un sistema de adquisición de datos}

El mundo real a escala macroscópica se suele comportar de forma analógica, ya que las magnitudes físicas varían de forma continua. Sin embargo, la mayoría de los sistemas de control modernos disponen de un procesador digital y en muchos casos un microordenador (PC).

Un sistema de adquisición de datos y de control comprende los sistemas y dispositivos de la siguiente figura. Fig. 1

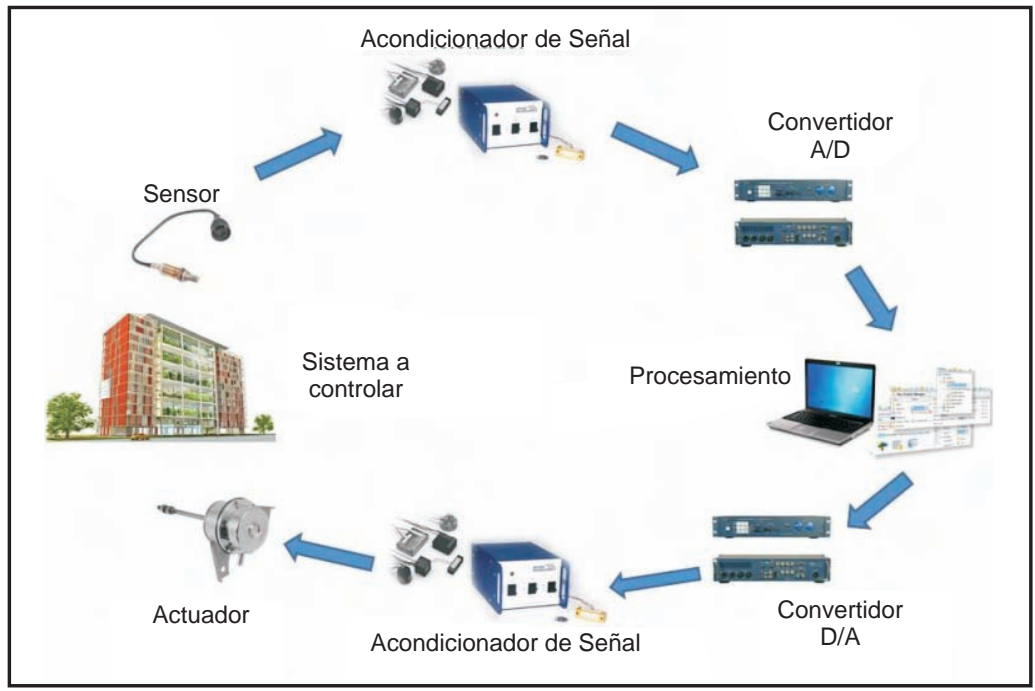

Fig.1 Componente de un sistema SCADA. 
El primer elemento es el sensor, captador o transductor de entrada. Es un dispositivo que transfiere energía del sistema que se analiza y controla, denominado EDIFICIO, transformando la magnitud física que se mide (Corriente, voltaje, temperatura, presión, posición) en señales eléctricas pequeñas.

En general un Sensor está conectado a un circuito que genera una señal eléctrica de salida. Esta señal tiene frecuentemente características inadecuadas para su ulterior uso, de modo que se debe de recurrir a la utilización de un Acondicionador de Señal cuya misión es adecuarla a las características de las etapas sucesivas 0 mejorar su calidad.

Puesto que la mayoría de los sensores proporcionan señales analógicas, éstas necesitan ser convertidas en digitales para su posterior utilización. Esta función la realiza un convertidor analógico-digital A/D.

Los datos digitales se utilizan en el procesador digital para que, de acuerdo con su programación, se realice alguna de las siguientes operaciones: Procesado, almacenamiento, representación y transmisión. La señal procesada, si resulta necesario, puede volver a ser utilizada en el mundo real, de modo que es precisa su reconversión en analógica a, dicha función la cumple el convertidor digitalanalógico D/A.

A su salida, otro acondicionador la adecúa a las necesidades específicas del elemento actuador o transductor de salida.

\section{Localización del sistema SCADA}

La implementación del sistema SCADA será dentro de los predios de la Universidad Nacional Autónoma de Honduras, UNAH, Fig. 2, específicamente en cada uno de sus edificios, y así obtener la adquisición y almacenamiento de datos, para recoger, procesar y almacenar la información recibida en forma continua y confiable. La instalación de cada uno de los equipos instalados (PC con tarjeta de adquisición de datos, PLC, CONTACTORES) estarán situados en lugares estratégicos del área geografía, recibiendo las señales de los sensores de movimiento y sensores capacitivos, que comandan los elementos finales de control, ejecutando el software de la aplicación SCADAque en nuestro caso es Labview, de National Instruments.

Existe equipos previamente instalados por personal de la Dirección Ejecutiva de Gestión de Tecnología, DEGT (Switch, Patch Panel, Closet para equipo etc.) los cuales son los encargados de la transmisión de datos, donde éstos son propiedad 
de la universidad. Estos equipos ya montados hacen mucho más fácil la interconexión de cada edificio ya que están conectadas al servidor principal que se encuentra ubicado en el edificio Administrativo, en el CRA.

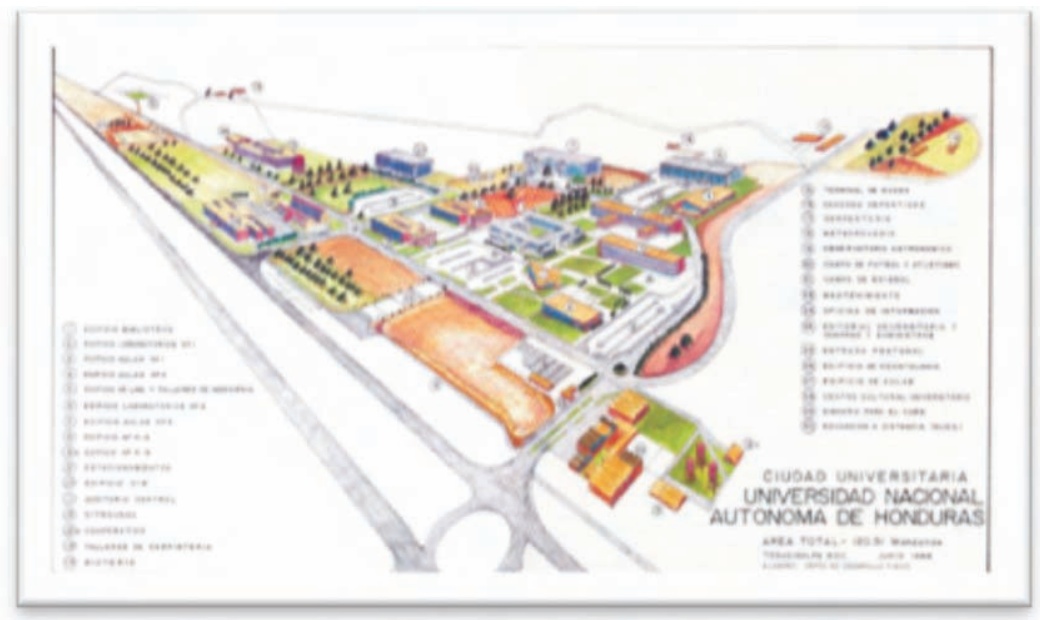

\section{Red de fibra óptica}

Actualmente la Universidad, cuenta con un Anillo de Fibra Óptica, el cual reúne todos los requisitos para la implementación de nuestro Sistema SCADA. Fig. 3. Esta Red es administrada por medio de la Dirección Ejecutiva de Gestión de Tecnología "DEGT" la cual, cuenta con presencia en todos los edificios de la UNAH.

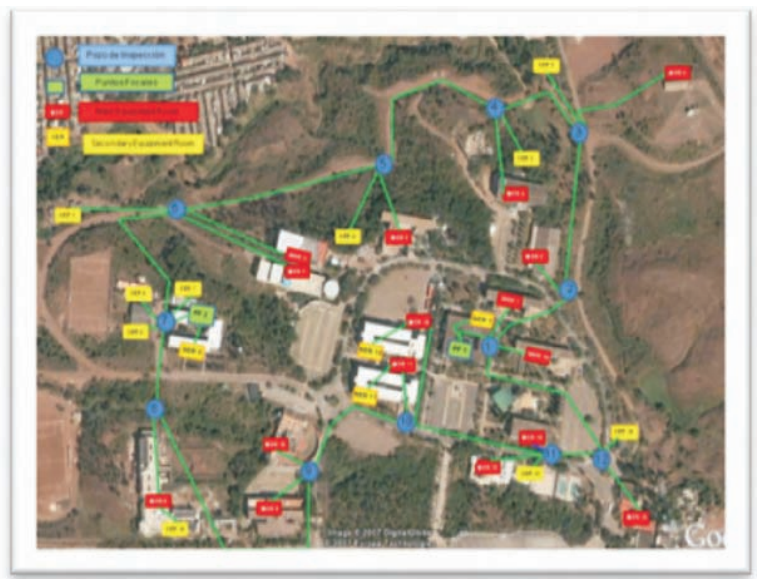

Fig. 3. Red de fibra óptica 


\section{PROTOTIPO DE SCADA}

\section{Introducción}

A continuación se presenta el programa Labview, y la tarjeta de adquisición de datos DAQ USB 6008, Fig. 4, donde logramos adquirir datos de variables físicas y también controlar un actuador luminoso.

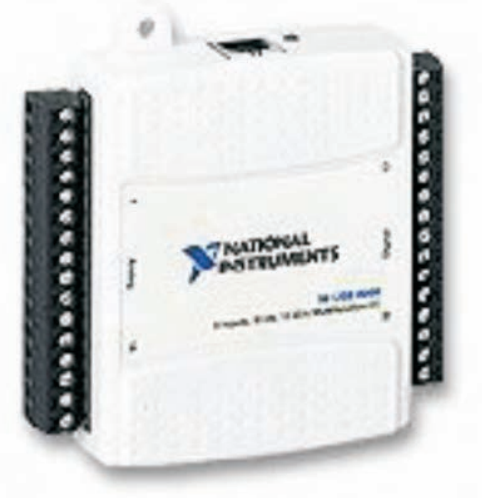

Fig. 4 Tarjeta de adquisición de datos

Es importante recordar que Lab-VIEW es un software gráfico de test para control y diseño mediante la programación. Los programas diseñados con Lab-View se llaman VI (Virtual Instrument)

\section{Principales usos de Labview}
a) Adquisición de datos
b) Control de instrumentos
c) Automatización industrial o PAC
d) Controlador de automatización programable
e) Diseño de control: prototipaje rápido y hardware-enel-bucle (HIL)

\section{Montaje del experimento}

Nuestro objetivo principal, es desarrollar una plataforma de SCADA de bajo costo con el fin de evidenciar y demostrar que este tipo de sistemas y tecnología la puede aplicar la UNAH. 


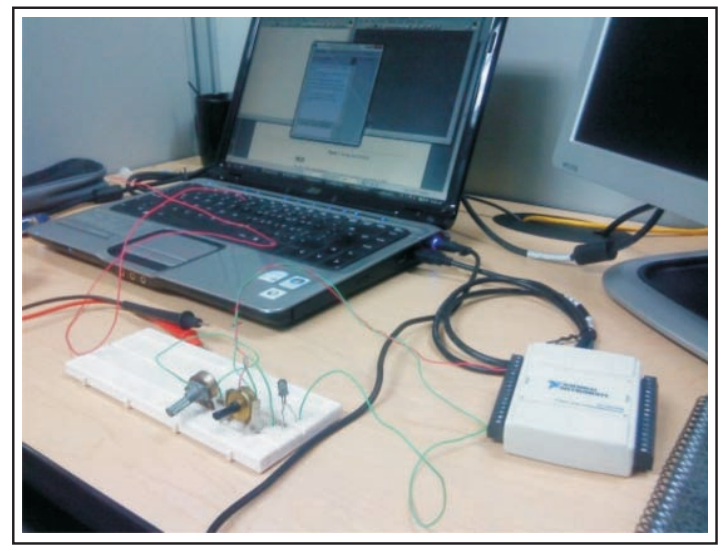

Fig. 5. Montaje.

\section{Procedimiento experimental}

Con este circuito, simulamos con el potenciómetro de 2KÙ un sensor, donde al variar la resistencia variamos el voltaje de entrada de la tarjeta DAQ, y así nuestra DAQ recoge esta señal y la envía a la computadora; el programa Lab-View procesa esta señal y dependiendo de la señal adquirida puede tomar decisiones preprogramadas, realizando acciones como de enviar una señal de voltaje al LED, el cual simula una lámpara que se enciende y se apaga según la señal que envía el Potenciómetro. Fig 5.

También se tiene control de las señales enviadas de la DAQ 6008 con el programa Lab-View. Con este software si se requiere se puede cambiar el tipo de señal enviada que controla el LED para su control o maniobra.

\section{ANÁLISIS FINANCIERO}

Aquí determinamos la viabilidad económico-financiera para implementar el proyecto SCADAen laUNAH.

\section{Fuentes de financiamiento}

Del total de la inversión requerida para iniciar operaciones, el 30\% será aportado por algún organismo Internacional, el Estado o la UNAH y para el 70\% restante se obtendrá por medio de un financiamiento. El financiamiento se obtendrá en la banca privada mediante un préstamo, 5 años plazo, 18 meses de gracia y pagos trimestrales de capital e intereses. Fig. 6. 


\begin{tabular}{|c|c|c|c|c|c|c|c|}
\hline \multicolumn{5}{|c|}{$\begin{array}{c}\text { Financiamiento por medio del BCIE } \\
5 \text { años plazo, } 18 \text { meses de gracia y pagos trimestrales de Capital e Intereses }\end{array}$} & \multirow{2}{*}{$\begin{array}{l}\text { 음 } \\
\text { 음 } \\
\text { 뜸 }\end{array}$} & \multirow{3}{*}{$\frac{\frac{1}{5}}{\frac{\cos }{\sigma}}$} & \multirow{3}{*}{$\begin{array}{l}\text { 岃 } \\
\text { 㟧 } \\
\text { 点 } \\
\geqq\end{array}$} \\
\hline MONTO: 2 & $32,620.63$ & TAS & $10 \%$ & & & & \\
\hline \multicolumn{5}{|c|}{ * Valores en Lempiras } & & & \\
\hline PERÍODO & CUOTA & CAPITAL & INTERESES & SALDO & \multirow{3}{*}{ io } & \multirow{3}{*}{ 용 } & \multirow{3}{*}{$\begin{array}{l}\text { 。̊ } \\
\text { త్ } \\
\text { స్ } \\
\text { స్ }\end{array}$} \\
\hline 0 & & & & $2,732,620.63$ & & & \\
\hline 1 & $68,315.52$ & 0.00 & $68,315.52$ & $2,732,620.63$ & & & \\
\hline 2 & $68,315.52$ & 0.00 & $68,315.52$ & $2,732,620.63$ & \multirow{5}{*}{ ĩ } & \multirow{5}{*}{ 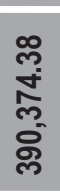 } & \multirow{5}{*}{ 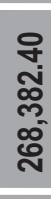 } \\
\hline 3 & $68,315.52$ & 0.00 & $68,315.52$ & $2,732,620.63$ & & & \\
\hline 4 & $68,315.52$ & 0.00 & $68,315.52$ & $2,732,620.63$ & & & \\
\hline 5 & $68,315.52$ & 0.00 & $68,315.52$ & $2,732,620.63$ & & & \\
\hline 6 & $68,315.52$ & 0.00 & $68,315.52$ & $2,732,620.63$ & & & \\
\hline 7 & $263,502.71$ & $195,187.19$ & $68,315.52$ & $2,537,433.44$ & \multirow{5}{*}{ io } & \multirow{5}{*}{ 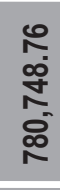 } & \multirow{5}{*}{ 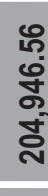 } \\
\hline 8 & $258,623.03$ & $195,187.19$ & $63,435.84$ & $2,342,246.25$ & & & \\
\hline 9 & $253,743.35$ & $195,187.19$ & $58,556.16$ & $2,147,059.06$ & & & \\
\hline 10 & $248,863.67$ & $195,187.19$ & $53,676.48$ & $1,951,871.87$ & & & \\
\hline 11 & $243,983.99$ & $195,187.19$ & $48,796.80$ & $1,756,684.68$ & & & \\
\hline 12 & $239,104.31$ & $195,187.19$ & $43,917.12$ & $1,561,497.49$ & \multirow{4}{*}{ ì } & \multirow{4}{*}{ 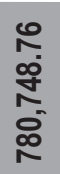 } & \multirow{4}{*}{ 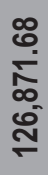 } \\
\hline 13 & $234,224.63$ & $195,187.19$ & $39,037.44$ & $1,366,310.30$ & & & \\
\hline 14 & $229,344.95$ & $195,187.19$ & $34,157.76$ & 1,171,123.11 & & & \\
\hline 15 & $224,465.27$ & $195,187.19$ & $29,278.08$ & $975,935.92$ & & & \\
\hline 16 & $219,585.59$ & $195,187.19$ & $24,398.40$ & $780,748.73$ & \multirow{5}{*}{$\begin{array}{l}n \\
0 \\
i \\
2\end{array}$} & \multirow{5}{*}{ 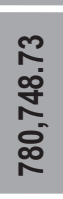 } & \multirow{5}{*}{ 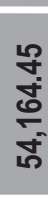 } \\
\hline 17 & $214,705.91$ & $195,187.19$ & $19,518.72$ & $585,561.54$ & & & \\
\hline 18 & $209,826.23$ & $195,187.19$ & $14,639.04$ & $390,374.35$ & & & \\
\hline 19 & $204,946.55$ & $195,187.19$ & $9,759.36$ & $195,187.16$ & & & \\
\hline 20 & $205,434.49$ & $195,187.19$ & $10,247.33$ & 0.00 & & & \\
\hline TOTAL & $3,660,247.80$ & $2,732,620.63$ & $927,627.17$ & & & & \\
\hline
\end{tabular}

Fig. 6. Plan de pago de préstamo.

\section{Ahorro en iluminación}

El estudio se realizó tomando en cuenta un análisis realizado con luminarias de 2x40 Watts, con una cantidad promedio, por edificio de 664 luminarias asumiendo 8 horas diarias de consumo.

Nuestra propuesta es ahorrar una hora de energía. En otras palabras reducir a 7 horas consumo actual suponiendo 8 horas. Fig. 7. 


\begin{tabular}{|l|r|r|r|r|r|}
\hline RESUMEN & \multicolumn{1}{|c|}{ Año 1 } & \multicolumn{1}{c|}{ Año 2 } & \multicolumn{1}{c|}{ Año 3 } & \multicolumn{1}{c|}{ Año 4 } & \multicolumn{1}{c|}{ Año 5 } \\
\hline Ahorro & $2,000,764.80$ & $2,100,803$ & $2,205,843$ & $2,316,135$ & $2,431,942$ \\
\hline Sueldos & 315000 & 346500 & 381150 & 419265 & 461191.5 \\
\hline Financiamiento & $273,262.08$ & 658,756 & 985,695 & 907,620 & $834,913.10$ \\
\hline Beneficiario & $1,412,502.70$ & $1,095,546$ & 838.997 & 989,249 & $1,135,837$ \\
\hline
\end{tabular}

Fig. 7. Ahorro de iluminación para 25 edificios.

Al final tenemos una relación costo y ahorro para 25 años. Fig.8.

\begin{tabular}{|l|r|r|r|r|}
\hline & $\begin{array}{c}\text { Consumo } \\
\text { trimestral } \\
(\mathbf{w})\end{array}$ & $\begin{array}{c}\text { Costo } \\
\text { de } \mathbf{k w}\end{array}$ & $\begin{array}{c}\text { Consumo } \\
\text { trimestral } \\
\text { (Lps) }\end{array}$ & Consumo anual \\
\hline Actual & $1,075,680$ & 3.72 & $4,001,529.60$ & $16,006,118.40$ \\
\hline Con proyecto & 941,220 & 3.72 & $3,501,338.40$ & $14,005,353.60$ \\
\hline AHORRO & 134,460 & & $500,191.20$ & $2,000,764.80$ \\
\hline
\end{tabular}

Fig. 8. Relación costo y ahorro

\section{TIRy VAN}

TIR: En la Fig. 9 A, se puede observar que la TIR para este proyecto es de $16 \%$, por lo que, bajo las condiciones planteadas anteriormente, éste resulta factible.

VAN: El valor presente neto (VPN) representa la sumatoria de todos los flujos de efectivo. Los flujos que se utilizan para calcular el VPN son L.223,370.09, demostrando que de acuerdo a lo establecido anteriormente este proyecto es factible. 


\begin{tabular}{|c|c|c|c|}
\hline \multicolumn{2}{|r|}{ TIR } & \multicolumn{2}{|c|}{ VAN } \\
\hline 0 & $-3660,247.80$ & 0 & $-3660,247.80$ \\
\hline 1 & $1,412,502.72$ & 1 & $1,412,502.72$ \\
\hline 2 & $1,095,546.26$ & 2 & $1,095,546.26$ \\
\hline 3 & 838.997 .87 & 3 & 838.997.87 \\
\hline 4 & $989,249.91$ & 4 & $989,249.91$ \\
\hline 5 & $1,135,837.44$ & 5 & $1,135,837.44$ \\
\hline & $16 \%$ & & PS. 223,370.09 \\
\hline
\end{tabular}

Fig. 9. A) La tasa interna de retorno y B) El valor presente neto.

\section{CONCLUSIONES}

1. El plan de inversión para los 25 edificios es L.3, 903,743.75, con un $30 \%$ de fondos propios o provenientes de fuentes externas L.1, 171,123.13 y un $70 \%$ financiado por una 0 varias entidades financieras L.2,732,620.63. Estas inversiones incluyen mobiliario, software y hardware únicamente. Los costos pueden disminuir considerando que el mobiliario o hardware que se requiere ya se tienen como parte de la red de fibra óptica administrada por la DEGT; se concluye que el detalle sea revisado por ellos y que sea la DEGT quien defina el costo final.

2. En las condiciones en que se plantea el proyecto, nos permite recuperar la inversión en un año y medio, si las utilidades o beneficios se aportan al capital para reducir intereses, como muestra de forma paralela la relación que existe entre el beneficio y el costo de la implementación del proyecto, donde el beneficio es muy rentable para más de cinco años. El proyecto es económicamente factible ya que nos da como resultado una tasa interna de retorno TIR del $16 \%$, y un valor presente del proyecto VAN de L. 223,370.09 para cinco años. 
3. De implementarse el proyecto de forma completa, el ahorro económico que tendría la UNAH seria más de un millón de lempiras anualmente con tan sólo disminuir una hora de iluminación. El estudio se realizó enfocado en el consumo de iluminación, haciendo falta investigar el consumo por aires acondicionados, centros de cocina, u otras cargas de alto consumo que posea la UNAH, que podrían ser controlados por el sistema SCADAy reducir aún más consumo.

\section{RECOMENDACIONES}

1. Entre las aportaciones más importantes del proyecto está que será una de las aplicaciones de la red de fibra óptica existente en la UNAH, por lo cual recomendamos que sea la DEGT la entidad que gestione y administre la adquisición de los fondos para la implementación del proyecto SCADA, arrancando con un edificio piloto, por ejemplo, el de Registro que es un edificio "semiinteligente". Asimismo, se podría seleccionar el de Derecho que ya cuenta con temporizadores.

2. Es sumamente importante contratar a un profesional de la ingeniería que labore en la DEGT, con dedicación exclusiva y amplios conocimientos de electricidad, software, autómatas y redes, otorgándole facultades suficientes para coordinar y gestionar la implementación y desarrollo de este proyecto y de los restantes proyectos de ahorro energético en los edificios de la UNAH, donde el costo de salario ya está incluido en los costo del proyecto. Sin este personal dedicado a tiempo completo será muy difícil llevar a cabo el proyecto.

\section{BIBLIOGRAFÍA}

Balcells,Josep y José Luis Romeral. Autómatas Programables. 1r. ed. Serie Mundo Electrónico. Barcelona: Marcombro, S.A., Boixareu; 1997.

Comer, Douglas E. Internetworking with tcp/ip. 5th ed. Massachusetts: Prentice Hall; 2006. Vol 1.

Manuales National Instruments. [Documento electrónico] Disponible en:

\section{http://www.ni.com/manuals/esal}

NI USB-6008/6009 User Guide and Specifications. [Documento electrónico] Disponible en:

\section{http://www.ni.com/pdf/manuals/371303I.pdff}

Olsson, Gustaff and Piani, Gianguido. Computer systems for automation and control. $1^{\circ}$ ed. New York: Prentice Hall; 1992.

Peterson, James L. y Silberschatz, Abraham. Sistemas Operativos. Conceptos Fundamentales. $2^{\circ}$ ed. Barcelona: Reverté S.A.; 1993. 